\title{
Multimedia Video Streaming using Advanced Compression and Bandwidth Efficient Technique
}

\author{
S D N Hayath Ali, M.Giri
}

\begin{abstract}
Multimedia is a modern way of communication where lots of images and videos are been transmitted over the wireless sensor network, hence managing the transmission of these records plays an important role when it comes to speed in which the multimedia transferred. In this paper compression technique and bandwidth utilization method is used for the efficient data transmission. Using this one can minimize the bandwidth utilization by compressing the video to the best format where packet won't be lost and complete packet will be transferred to the destination node. Remote sensor systems is a developing class of exceptionally powerful, complex system condition over which a wide scope of uses, for example, living space observing, object following, exactness farming, building checking and military frameworks are assembled. [1]The ongoing applications frequently produce earnest information and one-time occasion notices that should be conveyed dependably. The fruitful conveyance of such data directly affects the general execution of the framework. Solid correspondence is significant for sensor systems. In WSN's for viable transmission of still pictures, sound, and video data implements thorough necessities on the vitality utilization and throughput. [2] It is given in this to examine the presentation of steering traffic for interactive media traffic in WSN for multi jump nodes from the sink.
\end{abstract}

Keywords : Bandwidth efficiency, Multimedia, Nodes, video streaming, WSN

\section{INTRODUCTION}

The remote sensor organize is one of the most encouraging advances which will make our general public protected, secure, and comfortable[1]. A WSN as a social framework would convey both critical and non-dire data, which evidently ought not be dealt with similarly. The dire data, in territories like security, debacle, natural, and imperative conditions checking applications, must be brought through a WSN with higher unwavering quality and lower delay than other intermittent non-critical data for standard observing and working space control. It implies that a WSN must be fit for separating and organizing parcels relying upon their criticalness and significance as indicated by demands from

Manuscript published on November 30, 2019.

* Correspondence Author

SDN Hayath Ali*, Dept. of Computer Science, SGT College, Ballari, Karnataka, India. Email: hayath.ali@rediffmail.com

M.Giri, Dept. of CSE, Joginpally BR Engineering College, Hyderabad, India. Email: prof.m.giri@gmail.com

(C) The Authors. Published by Blue Eyes Intelligence Engineering and Sciences Publication (BEIESP). This is an open access article under the CC-BY-NC-ND license http://creativecommons.org/licenses/by-nc-nd/4.0/ the application layer. Fundamental propelling situation for this idea is the acknowledgment of value empowered systems for natural checking in catastrophe counteractive action and crisis reaction situations, for example, seismic tremors, underground mines and so on military surveillance and observation, for look and salvage in crisis circumstances, for condition based support and procedure control in industrial facilities, for foundation wellbeing checking in structures and in homes to acknowledge shrewd homes. The volume of sight and sound tangible information in nature surpasses that of old style scalar tactile information on a size of extents. For WMSNs with which the shortage of transmission capacity is standard, transmitting huge volume of mixed media information is a serious obstruction to survive. As a matter of first importance, techniques to decrease the measure of information to be transmitted are essential so as to chop down transfer speed necessity. At that point systems to boost the use of the accessible transfer speed must be investigated. On the off chance that conceivable, WMSNs need to receive advances that can possibly give higher accessible data transmissions. Information driven conventions are a significant part in various directing conventions in remote sensor systems [3]. Numerous fruitful steering conventions are displayed for WSNs and WMSNs until now. Coordinated Diffusion [4] and Turn [5] are two well known steering conventions for WSNs, which have gotten consideration. In the two conventions, demands are spread in arrange and steering is done dependent on information type. Each of the previously mentioned conventions is improved numerous times, as they are known as family Turn has numerous streams; for instance it isn't versatile, it isn't vitality proficient and so forth. Remote sight and sound sensor systems steering conventions are isolated in various manner [12].

\section{OVERVIEW OF COMPRESSION CLUSTERING}

In our strategy, sensor nodes are sorted out into bunches, and each group has a bunch head, spoke to by the strong square. Sensor nodes in each bunch transmit their unique information to the $\mathrm{CH}$ without utilizing $\mathrm{CS}$. We accept each $\mathrm{CH}$ knows the projection vectors of all nodes inside its bunch. In genuine frameworks, the estimation coefficient ij can be produced utilizing a pseudorandom number generator seeded with the identifier of the node vj. Consequently, given the identifiers of the nodes in the system, the estimation grid can be effectively built at CHs or the sink locally. For applications like security reconnaissance and traffic observing, practicality is key in the conveyance of the caught video information. Any miss of cut-off time implies framework disappointment and can cause security break or traffic occurrences.

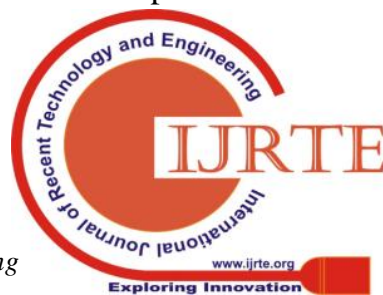


Make an interpretation of this into arrange tasks, the implications could be twofold. On one hand, MAC conventions can use prioritization and administration separation plans to give constant video information higher need for prior access of the remote medium, or award it with access to better channels.

\section{ANALYSIS OF THE ADVANCED ROCHET SCHEME}

In our technique, sensor nodes are sorted out into bunches, and each group has a bunch head, spoke to by the strong square. Sensor nodes in each group transmit their unique information to the $\mathrm{CH}$ without utilizing $\mathrm{CS}$. We accept each $\mathrm{CH}$ knows the projection vectors of all nodes inside its group. In genuine frameworks, the estimation coefficient ij can be produced utilizing a pseudorandom number generator seeded with the identifier of the node vj. In this manner, given the identifiers of the nodes in the system, the estimation lattice can be effectively built at CHs or the sink locally. For applications like security reconnaissance and traffic observing, practicality is key in the conveyance of the caught video information. Any miss of cut off time implies framework disappointment and can cause security break or traffic episodes. Make an interpretation of this into organize conventions can use prioritization and administration separation plans to give continuous video information higher as information transmission by methods for remote system has gotten broad and exceptionally urgent the need of media traffic recuperation framework has become a required errand. Various inquire about works have been done distinguishing creative plans to advance better video preparing gushing. To ensure constant video information against channel misfortunes a few establishment codes, for example, luby change, raptor and online codes are commonly received as the application layer FEC plot because of its high coding effectiveness, low handling time, and solid blunder revision ability. The progressed ROCHET plan will make the information to be compacted by the group head and course the video over the system from the source to the goal, the association between the bunches will be finished by cluster head itself. Because of bunching vitality is overseen in better manner and because of pressure method video size can be decreased. Transfer speed for a way for the transmission is fixed for any bundle transmission reason, if the size of the video is diminished to the base sum one can ready to transmit the video without putting pressure on the system. need for prior access of the remote medium, or award it with access to better channels.

\section{ADVANCED ROCHET ALGORITHM}

With restricted transfer speed accessible, having WMSNs to convey the entirety of the caught sight and sound information is surely not financially savvy. Subsequently, it is required to acquaint ways with decrease the volume of information to be transmitted. For instance over the top repetitive information can be expelled by applying installed nearby preparing calculations, or by executing appropriated source coding and interactive media in-arrange handling among neighboring nodes. In the mean time, conceivable in-organize information stockpiling arrangements can likewise be utilized to confine the need of information transmissions. tasks, the implications could be twofold. On one hand, MAC

\section{A. Algorithm}

Step 1: Create cluster of nodes across the area as cl1. Clustering(c1) // Here clustering is created

Step 2: Initializing the nodes with energy for working purpose. Ne.

Node(ni)=Random $(10,20) / /$ Nodes are initialized with some random initial energy.

Step 3: Selecting the cluster head by comaring the highest energy level of all the nodes with $\mathrm{CHi}$.

ClusterHead(ni)=Energy(ni) // The highest energy of the nodes are considered as the clusterHead.

Step 4: Collect the video data by the cluster head and compress it using the video encoder technique to minimize the content size.

Step 5: Select a shortest path for the data transmission from source to destination and transmit the data.

\section{RESULTS AND DISCUSSIONS}

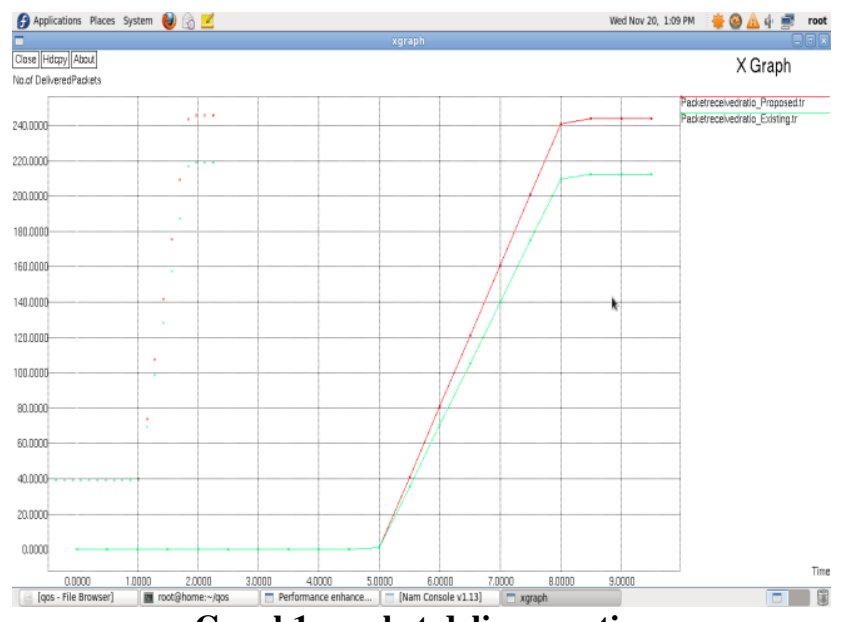

Graph1: packet delivery ration.

The above graph gives the comparison of the existing and proposed system with respect to the packet delivery from source to destination, as one can notice that the proposed system is almost $10 \%$ better the existing system.

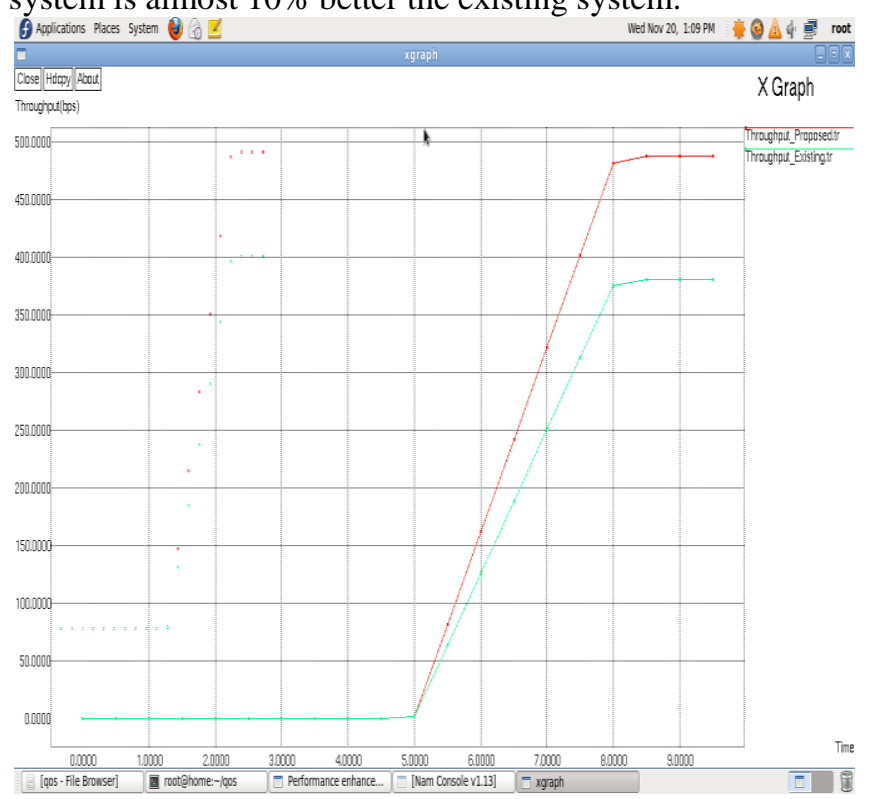

Graph2: Throughput.

hed By: 
The throughput of the proposed system is better than the existing system as the data is compressed, hence in less time more amounts of data is transmitted over the network.

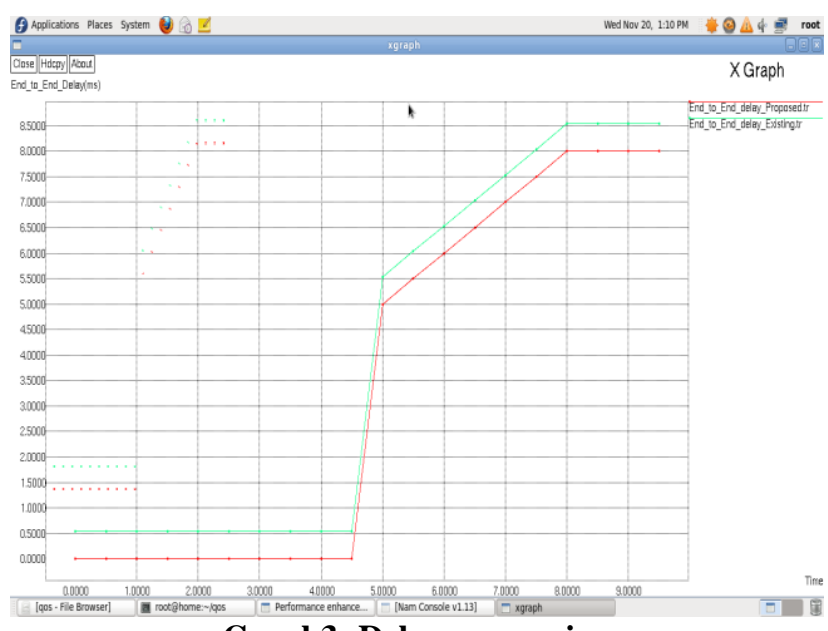

Graph3: Delay comparison.

The above graph is a delay comparisons of the existing and proposed system, as the network uses the clustering and compression technique with low bandwidth usage, delay is less in the proposed system.

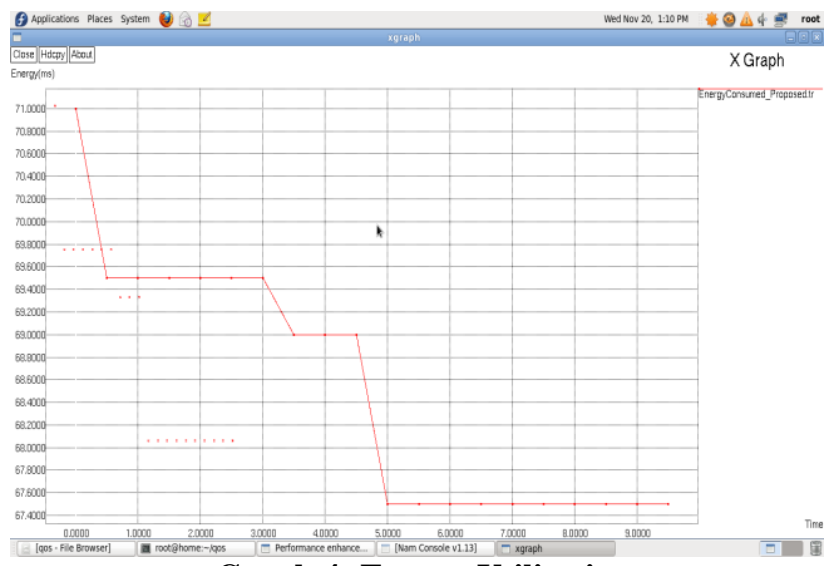

Graph 4: Energy Utilisation.

The energy requirement graph is given in above screen shot, as WSN is powered using the battery managing the power is one of the crucial thing in the data transmission, as we are using the clustering and compression, less work is needed by the sensor nodes hence energy is less utilised.

\section{a. TABLE OF CHART}

\begin{tabular}{|l|l|}
\hline No. of Nodes & 100 \\
\hline Area & 600 X 600 \\
\hline MAC & 802.11 \\
\hline Simulation Time & $50 \mathrm{sec}$ \\
\hline Traffic Source & CBR \\
\hline Rate & $100 \mathrm{~Kb}$ \\
\hline Propagation & Two Ray Ground \\
\hline Antenna & Omni Antenna \\
\hline Initial Energy & $8.1 \mathrm{~J}$ \\
\hline Transmission Power & 0.650 \\
\hline Receiving Power & 0.385 \\
\hline
\end{tabular}

The compression technique provide the efficient method for the data transmission over the wireless sensor network as the packets are compressed and the technique makes sure that all the packets are transmitted over the network without loosing any of the packets the proposed method is best for multimedia data transfer. Bandwidth are efficiently utilized hence lots of free bandwidth are available for other purpose also. The data on areas and appropriation of sensor nodes is utilized to plan the information assortment technique in bunch structure. Sensor nodes are composed into groups. Inside a bunch, information are gathered to the group heads by most brief way directing; at the bunch head, information are packed to the projections utilizing the CS method. The projections are sent to the sink following a spine tree. We initially proposed an expository model that reviews the connection between the size of groups and number of transmissions in the cross breed CS technique, to locate the ideal size of bunches that can prompt least number of transmissions. At that point, we proposed a brought together grouping calculation dependent on the outcomes acquired from the systematic model. WMSNs are the main thrust behind numerous interactive media applications in the time of Internet of things (IoT) because of their capacity to deliver sight and sound observation information including picture, video, and gushing media. Throughout the years, the examination network has gained a lot of ground toward the multiplication of WMSNs. Regardless, challenges still exist because of the asset imperatives and the one of a kind attributes of WMSNs. In this paper, we plot the attributes of WMSNs, and yet we distinguish the separately upheld prerequisites and for every particular necessity we summarize the current arrangement draws near.

\section{ACKNOWLEDGMENT}

The authors would like to thank a great support of

\section{REFERENCES}

1. E.M. Royer ,C.E. Perkins, "Especially named On-[1] and D. Culler, "An Analysis of a Large Scale Habitat Monitoring Application-Nov. 2004.

2. E. Candes and M. Wakin, "An Introduction to Compressive Sampling," IEEE Mar. 2008.

3. J. N Al-Karaki, "Routing Techniques in Wireless Sensor Networks: A Survey," 2004, pp. 6-28.

4. Intanagonwiwat, "Coordinated dispersion: A versatile and powerful correspondence worldview for sensor systems August 2000

5. W. R. Heinzelman" Adaptive conventions for Information Dissemination in Wireless Sensor Networks".

6. R. Baraniuk, "Compressive Sensing [Lecture Notes]," IEEE July 2007.

7. D. Donoho, "Packed Sensing,". 2006.

8. J. Haupt, W. Bajwa, M. Rabbat, and R. Nowak, "Packed Sensing for Networked Data," Mar. 2008.

C. Luo, F. Wu, Compressive Data Gathering for Large-Scale Wireless Sensor Networks," Proct. 2009. 


\section{AUTHORS PROFILE}

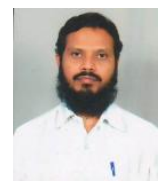

S Dada Noor Hayath Ali, Principal, Sree Guru Thipperudra College, Ballari, Karnataka. He pursued Bachelor of Science in Computer Science in 1999 from Sri Krishnadevaraya University, Anantapur, Andhra Pradesh, India. He did his Masters in Computer Science in 2001 from PVKK College, affiliated to Sri Krishnadevaraya

University, Anantapur.

He did his M.Phil in Computer Science in 2008 from Bharathiar University, Coimbatore, Tamil Nadu, India and now he is pursuing his Ph.D from the same university. Presently he is working in the department of Computer Science, SGT College, Ballari,an affiliated college of Vijayanagara Sri Krishnadevaraya University, Ballari, Karnataka. He has an overall 17 years of experience in the field of teaching, of which 3 years as corporate trainer. He has published paper in international journal and few more papers are in the pipeline for publication. His areas of interest include applications related streaming of videos on wired and Wireless Networks, Database Systems, System Programming and Data Mining.

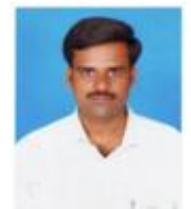

Dr.M.Giri, Prof.\& HOD, Dept. of CSE, Joginpally B.R.Engineering College, Hyderabad. He did his B.Tech in Computer Science \& Engineering from Sree Vidya Nikethan Engineering College, Tirupati, affiliated to JNTU, Hyderabad, in 2001. He did his M.Tech in Computer Science \& Engineering from School of IT, JNTU Hyderabad campus, Hyderabad in 2009. He did his Ph.D in Computer Science \& Engineering from Raalaseema University, Kurnool, in 2018. He is having 17 years of teaching experience. He organized and participated in various Workshops, FDPs, Seminars in different areas of Computer Science during his tenure. He has published 68 papers in various reputed International/National journals and Conferences. He is a member of IEEE, MCSIT, MIAENG and MCSTA. His research area includes Data Mining, Wireless Sensor Networks, Artificial Intelligence, Cryptography, Network Security, Cloud Computing and IoT. 